\title{
Differential Effects of Dual and Unihemispheric Motor Cortex Stimulation in Older Adults
}

\author{
Robert Lindenberg, ${ }^{\star}$ Laura Nachtigall, ${ }^{\star}$ Marcus Meinzer, Mira Maria Sieg, and Agnes Flöel \\ Department of Neurology, NeuroCure Cluster of Excellence, and Center for Stroke Research Berlin, Charité University Medicine, 10117 Berlin, Germany
}

\begin{abstract}
Bihemispheric transcranial direct current stimulation (tDCS) is thought to upregulate excitability of the primary motor cortex (M1) using anodal stimulation while concurrently downregulating contralateral M1 using cathodal stimulation. This "dual" tDCS method enhances motor learning in healthy subjects and facilitates motor recovery after stroke. However, its impact on motor system activity and connectivity remains unknown. Therefore, we assessed neural correlates of dual and unihemispheric anodal tDCS effects in 20 healthy older subjects in a randomized, sham-controlled study using a cross-over design. Participants underwent tDCS and simultaneous functional magnetic resonance imaging during a choice reaction time task and at rest. Diffusion tensor imaging (DTI) allowed us to relate potential functional changes to structural parameters. The resting-state analysis demonstrated that, compared with sham, both dual and anodal tDCS decreased connectivity of right hippocampus and M1 (contralateral to the anode position) while increasing connectivity in the left prefrontal cortex. Notably, dual but not anodal tDCS enhanced connectivity of the left dorsal posterior cingulate cortex. Furthermore, dual tDCS yielded stronger activations in bilateral M1 compared with anodal tDCS when participants used either their left or right hand during the motor task. The corresponding $\mathrm{tDCS}$-induced changes in laterality of activations were related to the microstructural status of transcallosal motor fibers. In conclusion, our results suggest that the impact of bihemispheric tDCS cannot be explained by mere add-on effects of anodal and concurrent cathodal stimulation, but rather by complex network modulations involving interhemispheric interactions and areas associated with motor control in the dorsal posterior cingulate cortex.
\end{abstract}

\section{Introduction}

Transcranial direct current stimulation (tDCS) is a noninvasive brain stimulation technique that has been successfully used to transiently modulate cortical excitability (Nitsche and Paulus, 2001) and induce long-lasting behavioral changes (Reis et al., 2009). Depending on its polarity, tDCS causes a depolarization (anode) or hyperpolarization (cathode) of neuronal membranes (Nitsche and Paulus, 2000) with after-effects that are mediated by calcium-dependent plasticity of the glutamatergic system (Nitsche et al., 2003). Performance-independent tDCS effects on the primary motor cortex (M1) have been studied extensively using electrophysiological parameters to quantify effect sizes and the time course of tDCS-induced changes (Nitsche and Paulus, 2011). In addition, functional magnetic resonance imaging (fMRI) at rest and during task performance has been used to investigate correlates of unihemispheric M1 stimulation (Baudewig et al., 2001; Stagg et al., 2009; Alon et al., 2011; Antal et

Received Jan. 6, 2013; revised March 28, 2013; accepted April 21, 2013.

Author contributions: R.L., M.M., and A.F. designed research; R.L., L.N., and M.M.S. performed research; R.L. and L.N. analyzed data; R.L., M.M., and A.F. wrote the paper.

This work was supported by the Deutsche Forschungsgemeinschaft Grants FI-379-8/1, 379-10/1, and DFG-Exc257 (A.F.), the Bundesministerium für Bildung und Forschung Grants FKZ0315673A (M.M.), 01 GY1144 (M.M.), and $01 E 00801$ (A.F.), and the Else Kröner-Fresenius Stiftung Grants 2009-141(A.F.) and 2011-119 (R.L.).

*R.L. and L.N. contributed equally to this work.

The authors declare no competing financial interests.

Correspondence should be addressed to either Dr Robert Lindenberg or Dr Agnes Flöel, Depart-

ment of Neurology, Charité University Medicine, Berlin, Charitéplatz 1, 10117 Berlin, Germany,

E-mail: robert.lindenberg@charite.de or agnes.floeel@charite.de.

DOI:10.1523/JNEUROSCI.0055-13.2013

Copyright $(2013$ the authors $\quad 0270-6474 / 13 / 339176-08 \$ 15.00 / 0$ al., 2011; Kwon and Jang, 2011; Polania et al., 2011b,c). Modulations of resting-state functional connectivity caused by tDCS appear to depend on the site of stimulation and other factors such as stimulation durations, making it virtually impossible to draw a conclusive overall picture so far due to the different designs used in previous studies. With regard to task-related activity, some studies did not detect significant tDCS-induced changes in M1 of either hemisphere during anodal or cathodal stimulation (Antal et al., 2011), whereas other studies demonstrated an increase in task-related activity as an after-effect of anodal stimulation (Stagg et al., 2009) or a decrease as an after-effect of cathodal stimulation (Baudewig et al., 2001). Remote effects in nonprimary motor areas and association cortices seem to be even more variable. However, small sample sizes and a wide spectrum of stimulation durations and intensities make it difficult to directly compare the results.

In addition to unihemispheric stimulation, bihemispheric or "dual" tDCS of left and right M1 has been described to enhance motor learning in healthy subjects (Vines et al., 2008) and to facilitate motor recovery in the chronic phase after stroke (Lindenberg et al., 2010, 2012a; Bolognini et al., 2011). This approach presumably upregulates M1 using anodal stimulation while downregulating the contralateral M1 with concurrent cathodal stimulation (Vines et al., 2008). However, direct comparisons of unihemispheric anodal versus dual stimulation are scarce, and the mechanisms underlying dual tDCS effects on motor system activity and functional connectivity have not yet been examined. Therefore, in this randomized sham-controlled study, we assessed neural correlates of bihemispheric and unihemispheric 
tDCS using a cross-over design. We hypothesized that both active tDCS conditions would exert similar effects on task-related and resting-state $\mathrm{AMRI}$ in the left hemisphere due to identical anode placements (upregulation of activity and strengthening of connections, compared with sham). In contrast, we expected more pronounced dual effects in the right hemisphere due to varied cathode placements (downregulation of activity and functional uncoupling). Because older individuals suffering from stroke or cognitive impairment are the main target group in present and future clinical tDCS trials, a group of healthy older adults was chosen.

\section{Materials and Methods}

Twenty healthy older subjects participated in this study (10 women; mean age \pm SD $68.2 \pm 5.0$ years; range $61-77$ ). They were all righthanded according to the Edinburgh inventory (Oldfield, 1971). None of the subjects reported use of psychoactive medication or recreational drugs, and none of them had a history of neurological or psychiatric disorders. Using the neuropsychological test battery of the Consortium to Establish a Registry for Alzheimer's Disease (CERAD-Plus; www. memoryclinic.ch), we assured normal cognitive functioning in all participants (scores within $\pm 1.5 \mathrm{SD}$ of the mean for the CERAD normative sample in all subtests).

In a crossover within-subjects design, subjects participated in three identical MRI sessions with concurrent dual, anodal or sham tDCS, separated by at least 1 week to prevent carry-over effects. The order of stimulation conditions was balanced across the group so that each order was used for 3-4 participants. The study was approved by the local ethics committee, conducted in accordance with the Declaration of Helsinki, and written informed consent was obtained from all participants.

Transcranial direct current stimulation. Direct current was delivered through a battery-driven MRI-compatible stimulator (DC-Stimulator Plus, NeuroConn) positioned outside the scanner room. Filter boxes (absorbing radio frequency noise) inside and outside the scanner room were placed between stimulator and electrodes, and cables were equipped with $5 \mathrm{k} \Omega$ resistors as described previously (Antal et al., 2011; Meinzer et al., 2012). The anode was inserted in a $5 \times 7 \mathrm{~cm}^{2}$ salinesoaked synthetic sponge and centered over left M1 according to position C3 of the 10-20 EEG system in all stimulation conditions. For dual stimulation, the cathode was placed over the right $\mathrm{M} 1$, according to position C4 (cf. Lindenberg et al., 2010). In the anodal condition, the cathode served as a reference electrode $\left(10 \times 10 \mathrm{~cm}^{2}\right)$ placed over the contralateral supraorbital region. In the sham condition, the electrode setup was pseudo-randomly assigned to participants (either "dual" or "anodal") and balanced across the group. Correct electrode placement was verified on the T1-weighted images in every subject. In all conditions the current was increased over $10 \mathrm{~s}$, eliciting an initial transient tingling sensation on the scalp. During sham stimulation, the current was turned off after $30 \mathrm{~s}$. During active stimulation, a current of $1 \mathrm{~mA}$ was constantly delivered for $30 \mathrm{~min}$ during resting-state and task-related fMRI. In the sham condition, fMRI commenced after the current was turned off. In between resting-state $(5 \mathrm{~min})$ and choice reaction time task (10 min), subjects participated in an overt semantic word-retrieval task (10 min). During the latter task, participants were presented with six categories and asked to generate 10 different exemplars for each category (for a detailed description, see Meinzer et al., 2012).

MRI parameters. All participants underwent MRI using a 3-tesla Siemens Trio MR-System at the Berlin Center for Advanced Neuroimaging. We used a $\mathrm{T}^{*}{ }^{*}$-sensitive echo-planar imaging sequence covering the whole brain for task-related BOLD fMRI $(\mathrm{TR} / \mathrm{TA}=2000 \mathrm{~ms}$, $\mathrm{TE}=30$ ms, flip angle: $90^{\circ}, 32$ transverse slices, gap: $0.75 \mathrm{~mm}$, interleaved acquisition, voxel size: $3 \times 3 \times 3 \mathrm{~mm}^{3} ; 306$ volumes), and for resting-state BOLD-fMRI (TR/TA $=2300 \mathrm{~ms}$, TE $=30 \mathrm{~ms}$, flip angle: $90^{\circ}, 34$ transverse slices, no gap, interleaved acquisition, acquisition matrix: $64 \times 64$, voxel size: $3 \times 3 \times 4 \mathrm{~mm}^{3}$; 150 volumes). A T1-weighted sequence was acquired to facilitate normalization $(\mathrm{TR} / \mathrm{TA}=1900 \mathrm{~ms}, \mathrm{TE}=2.52 \mathrm{~ms}$, 192 sagittal slices; isotropic voxels, size $1 \mathrm{~mm}^{3}$ ). An additional fluid attenuated inversion recovery sequence helped excluding structural ab- normalities in all participants. Finally, we obtained a diffusion tensor imaging (DTI) sequence to compare functional results with measures of structural connectivity $(\mathrm{TR}=7500 \mathrm{~ms}, \mathrm{TE}=86 \mathrm{~ms}, 61$ axial slices, voxel size: $2.3 \times 2.3 \times 2.3 \mathrm{~mm}^{3} ; 64$ directions with a $b$ value of $1000 \mathrm{~s} / \mathrm{mm}^{2}$ and $10 b 0$ ). Although fMRI and T1-weighted sequences were acquired de novo in all three sessions, subjects underwent DTI only during the sham condition to prevent potential tDCS-induced changes in DTI parameters.

Choice reaction time task. Similar to a previous study (Kloppel et al., 2007), subjects were presented with three different symbols in the center of the visual field and instructed to respond with button presses using either their right or left index fingers, or to withhold a response (no-go condition). The symbols carried no directional information (triangle, square, and hexagon). Symbols were shown for $1000 \mathrm{~ms}$, followed by a blank screen of variable duration. Each symbol was presented 52 times, and there were 52 null events (blank screen) interspersed among the visual cues. The order of events and interstimulus intervals (ISI) were randomized. We used a jittered ISI ranging between 2000 and $5600 \mathrm{~ms}$ with an exponential distribution of ISI durations (more short than long ISIs), resulting in a task duration of $604 \mathrm{~s}$ (i.e., 302 EPI volumes). Subjects practiced the task before entering the scanner room for $\sim 5 \mathrm{~min}$. We deliberately chose this simple visually cued choice reaction time task (Kloppel et al., 2007) to prevent potential tDCS-induced behavioral effects because we intended to detect activity changes caused by the stimulation that could not be explained by performance.

Task-related fMRI data analysis. For the analysis of task-related fMRI data, SPM8 (Wellcome Department of Imaging Neuroscience, London, UK) implemented in MATLAB (Mathworks) was used. Preprocessing comprised motion correction, spatial normalization, and spatial smoothing with a Gaussian kernel of $8 \mathrm{~mm}$ (FWHM). Before estimation of the statistical model a high-pass filter of $128 \mathrm{~s}$ was applied.

The effects of all three sessions were determined in a single event related-model at the individual subject level to account for sessionspecific effects. The design matrix for the statistical analysis comprised four covariates-of-interest (left tap, right tap, no-go, null) as well as covariates-of-no-interest (movement parameters). The onset of the regressors "left tap" and "right tap" was set to the button presses, whereas the onset of the regressor "no-go" was set to the stimulus presentation. Errors (omissions or false alarms) were modeled as additional regressors; therefore, the analysis comprised only correct trials. Contrasts of interest were estimated for each subject and session (left tap or right tap or no-go versus implicit baseline), and entered into a random effects group analysis with "subject" as random factor. Due to excessive movement one subject had to be excluded from the analysis.

In addition to whole brain statistics, we conducted region-of-interest (ROI) analyses using the Anatomy Toolbox (Eickhoff et al., 2005) implemented in SPM8. We extracted mean $\beta$ values of primary motor cortices (Brodmann area, BA 4p, which comprised the center of clusters associated with left and right tap) and premotor areas (BA 6) bilaterally for the three contrasts of interest in all three imaging sessions. Differences of contrast-specific $\beta$ values between sessions were analyzed using repeated-measure ANOVAs with post hoc pairwise comparisons (Bonferroni-corrected).

Even though the analysis of data acquired during the verbal fluency task was beyond the scope of this paper, we also assessed the impact of the two active stimulation conditions versus sham on M1 activity during this task using the same ROIs as above (for a detailed description, see Meinzer et al., 2012). This analysis explored whether potential effects of tDCS on motor activity during the language task may explain effects observed during the subsequent motor task (cf. Antal et al., 2007). Two subjects had to be excluded in this analysis due to movement artifacts during the overt word generation (the same subject excluded in the motor task and an additional subject).

Resting-state data analysis. We analyzed the resting-state fMRI data using eigenvector centrality mapping (ECM), an established graphtheory based approach implemented in LIPSIA (Lohmann et al., 2010). In short, ECM allows for a quantitative characterization of network structures by determining the interconnectedness of central brain regions ("hubs") across the entire brain without requiring any a priori 
assumptions (Bonacich, 2007). This characterization comprises eigenvector centrality values, which are assigned to each voxel according to its connection strength to other voxels central within a network; higher values indicate stronger connections (Lohmann et al., 2010; Taubert et al., 2011). In line with previous work from our group (Meinzer et al., 2012), we were specifically interested in low-frequency fluctuations, which have been shown to be valuable for assessing interregional dependencies (Salvador et al., 2005). Preprocessing steps included motion correction, spatial normalization to the Lipsia template (voxel size $3 \times 3 \times$ $3 \mathrm{~mm}^{3}$ ), bandpass filtering at $1 / 90 \mathrm{~s}$, and spatial smoothing with a Gaussian kernel of $6 \mathrm{~mm}$.

To specifically analyze gray matter structures, we used the binary mask of a study-specific template created with FMRIB Software Library (FSL) via segmentation of the T1-weighted images (Smith et al., 2004). Voxelwise spectral coherence analyses were conducted on a single subject level for frequency intervals of $0.03-0.06 \mathrm{~Hz}$ for the two active tDCS and sham conditions separately (Meinzer et al., 2012). After $z$-transformation of the resulting matrices to obtain a Gaussian normal distribution (Lohmann et al., 2010), the spectral bands were averaged on an individual basis, and the resulting mean images were compared between the three stimulation conditions by whole-brain pairwise $t$ tests. Clusters were considered significant at $p<0.05$, corrected for multiple-comparisons using a Monte-Carlo simulation (Lohmann et al., 2010).

Probabilistic tractography and correlations with $\mathrm{fMRI}$. FSL was used for preprocessing and fiber tracking (http://www.fmrib.ox.ac.uk/fsl). We applied a 3D affine registration to correct for eddy currents and head motion, performed brain extraction, and calculated a probability distribution of fiber directions for each voxel, allowing estimates of two directions per voxel (Behrens et al., 2007). Because we were specifically interested in connections between M1 hand areas, we chose an approach described previously (Wahl et al., 2007): cubic ROIs were drawn on the individual fractional anisotropy (FA) images in native diffusion space in the white matter underlying the hand knob, and connections between those ROIs were tracked from left to right and vice versa. Sagittal exclusion masks were placed along the midline dorsal to the corpus callosum and axial exclusion masks caudal to the corpus callosum. To test for specificity, we additionally reconstructed the pyramidal tract in both hemispheres using a seed region in the anterior pons as well as waypoint masks in the ipsilateral posterior limb of the internal capsule and the cubic subcortical white matter ROIs also used for tracing transcallosal connections (Lindenberg et al., 2012b; Ruber et al., 2012). The resulting probabilistic streamlines were constrained to voxels with $>10 \%$ of the individual tract-specific connection probability, and binarized to define tract masks. The masks of left-right and right-left streamlines were then merged to extract tract-specific FA in native space (only non-zero values) for correlation analyses of DTI and fMRI parameters.

Correlation between structure and function. To assess whether some of the variance in expected tDCS-induced modulations of fMRI activation patterns would be explained by the microstructural status of transcallosal motor fibers, we conducted correlation analyses between extracted FA values and the change in laterality indices (LI) of left and right tap. The following equation was used to calculate LI (Seghier, 2008): LI = (contralateral - ipsilateral) / (|contralateral $\mid+$ ipsilateral $\mid)$, where "contralateral" and "ipsilateral" refer to $\beta$ values extracted from M1 contralateral or ipsilateral to the moving hand. The resulting values range between -1 and 1 with positive values indicating stronger activation of the M1 contralateral to the moving hand, and negative values indicating stronger activation of the M1 ipsilateral to the moving hand. tDCSinduced changes in laterality were then defined as: $L I_{\text {dual }}-\mathrm{LI}_{\text {sham }}$ and $\mathrm{LI}_{\text {anodal }}-\mathrm{LI}_{\text {sham }}$ to perform the above correlation analyses.

\section{Results}

No adverse effects were reported by the subjects or observed by the investigators during or after the stimulation. Subjects were not able to differentiate between the active and sham stimulation conditions, as indicated by questionnaire.

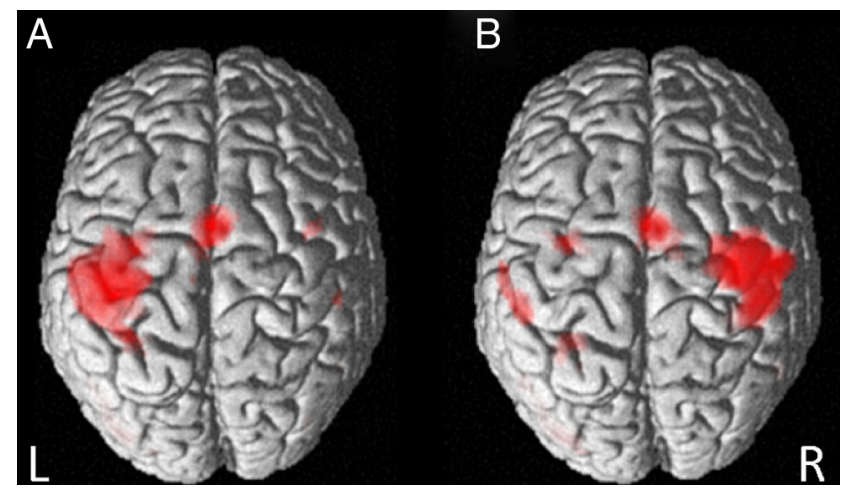

Figure 1. Whole brain conjunction analyses [dual $\cap$ anodal $\cap$ sham]. Results of the taskrelated analysis; surface renderings of clusters associated with $(\boldsymbol{A})$ right and $(\boldsymbol{B})$ left index finger tap compared with the implicit baseline (blank screen). Images are thresholded at $p<0.05$, corrected for family-wise error. L, Left; R, right hemisphere.

\section{Choice reaction time task: behavioral analysis}

Repeated-measure ANOVAs demonstrated that there was no effect of stimulation condition on the number of errors [left tap: $F_{(2,36)}=0.174, p=0.841$; tap right: $F_{(2,36)}=0.453, p=0.639$; no-go: $\left.F_{(2,36)}=1.481, p=0.241\right]$ or reaction times [left tap: $F_{(2,36)}=1.567, p=$ 0.223 ; right tap: $\left.F_{(2,36)}=0.889, p=0.420\right]$. These results are in line with previous studies that used simple motor tasks (Kloppel et al., 2007) and allowed us to investigate the neural correlates of stimulation without interaction with behavioral effects.

\section{Task-related fMRI}

The whole brain analyses revealed a highly consistent pattern of activation clusters across the three stimulation conditions (Fig. 1 ). We thus report the results of a conjunction analyses [dual $\cap$ anodal $\cap$ sham] in the following (Nichols et al., 2005). When subjects tapped with their right index fingers, clusters were found in left M1 and SMA as well as in the left fusiform gyrus. Left tap yielded most prominent clusters in the right M1 and SMA as well as additional left-hemisphere clusters in the inferior parietal lobule, fusiform gyrus, and dorsal premotor cortex (PMd). In the no-go condition, positive activation changes involved bilateral inferior and middle occipital gyri (all $p<0.05$, FWE-corrected on the voxel level). A more lenient threshold revealed additional clusters in the left SMA and inferior parietal lobule $(p<0.001$, uncorrected).

The ROI analysis (Fig. 2) demonstrated that cortical activations during right tap showed an effect of stimulation condition in right $\mathrm{BA} 4\left[F_{(2,36)}=4.505, p=0.018\right]$. Post hoc tests applying Bonferroni correction revealed a difference between dual and anodal stimulation with higher $\beta$ values during dual tDCS ( $p=$ $0.046)$, but no differences between dual and sham stimulation $(p=0.345)$ or anodal and sham stimulation $(p=0.415)$. In the left BA 4, no tDCS-induced differences were found when subjects tapped with their right index fingers $\left[F_{(2,36)}=2.157, p=0.130\right]$.

For left tap, an effect of stimulation became apparent in both right $\mathrm{BA} 4\left[F_{(2,36)}=5.478, p=0.008\right]$ and in left $\mathrm{BA} 4\left[F_{(2,36)}=\right.$ $3.711, p=0.034]$. Post hoc pairwise comparisons using Bonferroni correction demonstrated higher $\beta$ values of dual compared with anodal stimulation bilaterally (left BA $4, p=0.034$; right BA $4, p=0.008)$. No differences were found between $\beta$ values of anodal and sham stimulation in left BA $4(p=0.128)$ or right BA $4(p=0.197)$. Similarly, no differences between dual and sham stimulation became apparent in left BA $4(p=1.000)$ or right BA $4(p=0.632)$. 


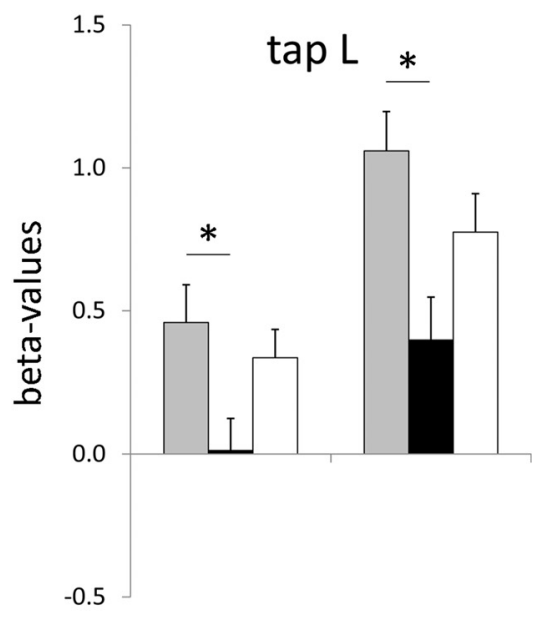

M1 L

M1 R
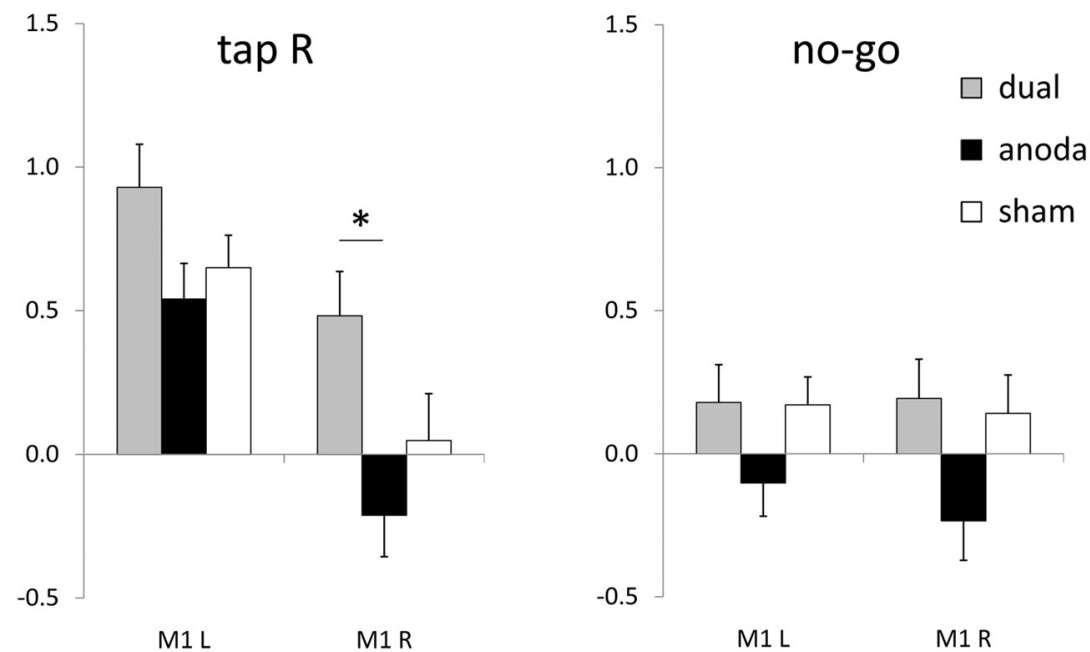

Figure 2. ROl analysis. Comparing task-related $\beta$ values of left index finger tap ("tap L"), right index finger tap ("tap R") and no-go during dual, anodal and sham tDCS.

Neither right tap (all $p>0.54$ ) nor left tap (all $p>0.05$ ) yielded significant differences between the stimulation conditions in left or right BA 6. Furthermore, no effect of stimulation was found in BA 4 or BA 6 in either hemisphere for the no-go condition (all $p>0.11$ ).

With regard to the word-retrieval task, no effect of stimulation was observed in BA 4 in either hemisphere (left: $F_{(2,34)}=0.372$, $p=0.692$; right: $\left.F_{(2,34)}=0.929, p=0.405\right)$ or BA 6 (left: $F_{(2,34)}=$ $1.339, p=0.276$; right: $\left.F_{(2,34)}=1.474, p=0.243\right)$. None of the post hoc comparisons yielded significant results (all $p>0.28$, Bonferroni-corrected).

\section{Association between DTI parameters and tDCS-induced functional changes}

Correlation analyses between FA values of transcallosal connections and tDCS-induced LI change yielded significant results for left tap (dual: $r=0.47, p=0.043$; anodal: $r=0.73, p<0.001$ ), whereas FA values did not correlate with LI changes during right tap (dual: $r=0.05, p=0.840$; anodal: $r=0.07, p=0.696$ ) (Fig. 3). tDCS-induced LI changes differed between dual and anodal conditions for right tap $\left(t_{(18)}=-2.98, p=0.008\right)$, but not for left tap $\left(t_{(18)}=0.50, p=0.620\right)$.

There were no significant correlations between LI changes and FA values of pyramidal tracts in either hemisphere. In more detail, LI change during dual tDCS did not correlate with FA of left (left tap: $r=0.039, p=0.875$; right tap: $r=0.021, p=0.731$ ) or right pyramidal tracts (left tap: $r=0.085, p=0.731$; right tap: $r=$ $-0.076, p=0.759)$. Similarly, anodal tDCS-induced LI change did not correlate with FA of left (left tap: $r=0.385, p=0.104$; right tap: $r=0.219, p=0.389$ ) or right pyramidal tracts (left tap: $r=0.301, p=0.210$; right tap: $r=0.186, p=0.445)$.

\section{Resting-state fMRI}

The resting-state data analysis demonstrated specific differences of low-frequency fluctuations between the three stimulation conditions. Comparing dual with sham stimulation, clusters of significantly higher ECM values were found in bilateral dorsolateral prefrontal cortices (DLPFC) and adjacent PMd, in the left dorsal posterior cingulate cortex ( $\mathrm{PPCC}$ ) and temporoparieto-occipital junction during dual tDCS. In contrast, dual tDCS induced a reduction of ECM values in the right frontal and parietal operculum, bilateral hippocampi/parahippocampal gyri and the cerebellum when compared with sham (Table 1; Fig. 4).
The comparison of anodal with sham stimulation yielded clusters of higher ECM values in the left supplementary motor are (SMA), left DLPFC (extending to the ipsilateral rostral frontal area), the left temporoparieto-occipital junction, and the right cuneus during unihemispheric anodal stimulation. Reduced ECM values were found in the right sensorimotor cortex, the left anterior/middle cingulate gyrus, right insula (extending to the ipsilateral parietal operculum), and bilaterally in the hippocampus and parahippocampal gyrus when anodal was compared with sham tDCS (Table 1; Fig. 4).

Finally, the comparison of dual and anodal tDCS revealed higher ECM values in the left $\mathrm{APCC}$ and adjacent SMA during dual stimulation and the vermis of the cerebellum during anodal stimulation (all $p<0.05$, Monte-Carlo corrected).

\section{Discussion}

This sham-controlled randomized study revealed differential effects of bihemispheric versus unihemispheric motor cortex stimulation on task-related and resting-state fMRI in healthy older adults. Independent of performance, task-related activity was stronger in bilateral M1 during dual compared with unihemispheric anodal tDCS when subjects tapped with left or right index fingers. tDCS-induced activity changes were partly explained by microstructural properties of transcallosal motor fibers, indicating the importance of interhemispheric interactions for tDCS effects. Furthermore, both active tDCS conditions counteracted the age-related lack of inhibition of the nondominant hemisphere during resting-state fMRI, whereas dual tDCS selectively modulated connectivity of the cingulate cortex.

\section{Task-related activity}

The main finding of our task-related analysis was more pronounced activity in bilateral M1 during dual compared with unihemispheric anodal tDCS. Those results were specific for M1, because tDCS did not yield significant changes in premotor cortices. We deliberately chose a simple task, in which performance was not modified by tDCS. If behavior had been influenced by tDCS, it would have been virtually impossible to disentangle tDCS and performance effects on BOLD signal changes. Based on electrophysiological investigations that suggest differential effects of anodal and cathodal tDCS on M1 excitability (MordilloMateos et al., 2012), concordant modulations of activity in 

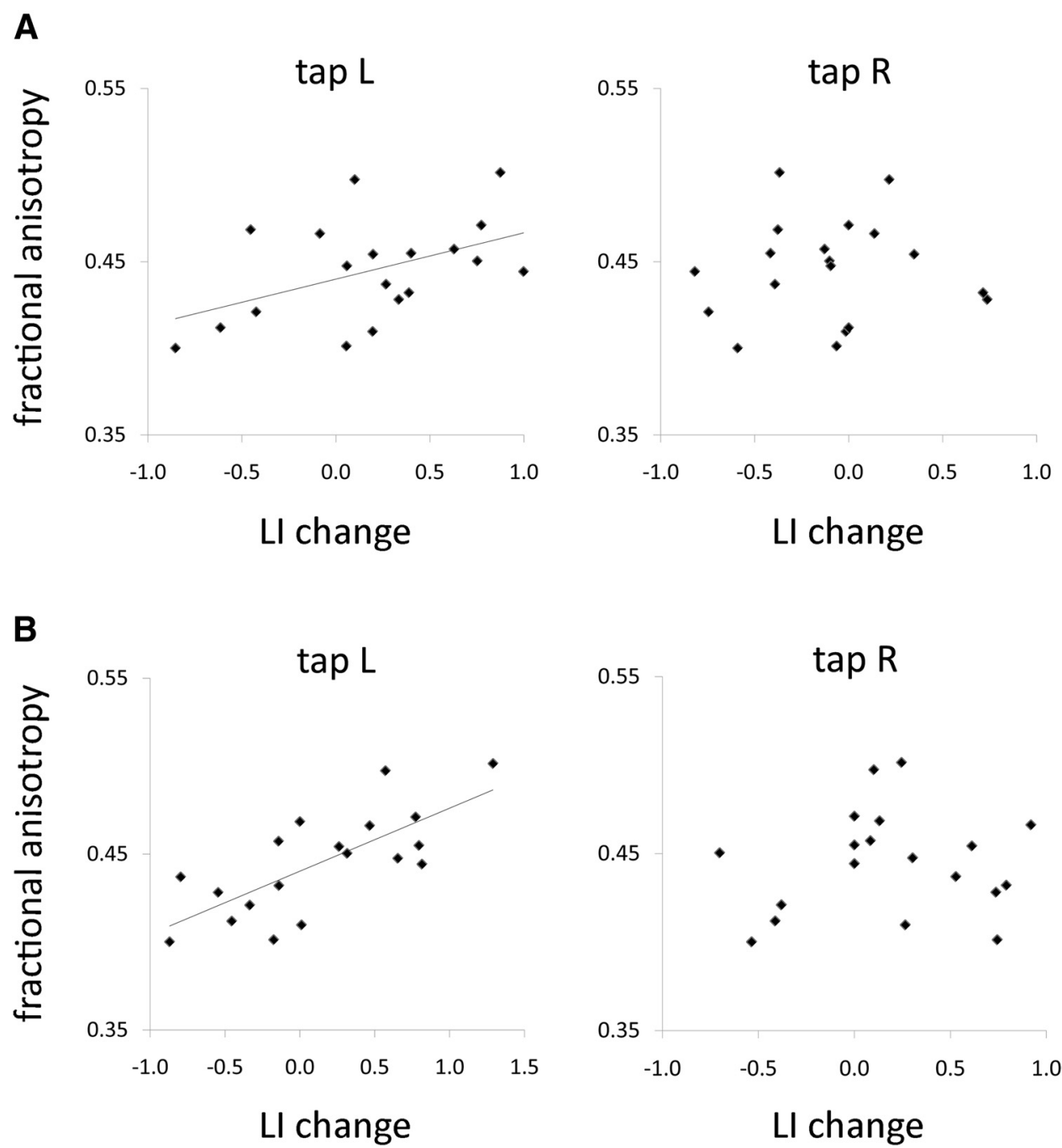

Figure 3. Correlations of laterality indices and fractional anisotropy. Correlating $\mathrm{FA}$ of transcallosal motor fibers and task-related laterality index changes induced by $(\boldsymbol{A})$ dual and $(\boldsymbol{B})$ anodal $\mathrm{tDCS}$. Tap L, Left index finger tap; tap R, right index finger tap.

bilateral M1 in our study seemed surprising at first glance. We had originally hypothesized that cathodal tDCS of right M1 (concurrently applied with anodal tDCS of left M1 in the dual setup) would yield a more focused pattern of brain activation in this area. However, our results suggest that dual tDCS not simply exerts mutually amplifying "anodal plus cathodal" effects. Likewise, because left M1 received anodal stimulation in both active tDCS conditions, differential effects of anodal and dual tDCS observed in left M1 cannot be merely explained by "direct" polarity-specific alterations of the cortex underneath the electrodes, but rather by modulations of interhemispheric interactions affecting bilateral M1. It should be noted in this context that the interpretation of task-related BOLD signal changes is challenging because both "activation" and "inhibition" can potentially yield BOLD signal increases (Lauritzen et al., 2012). As a complement to the task-related data, our resting state analysis (discussed in detail below) demonstrated connectivity increases in prefrontal areas ipsilateral to the anode and decreases in several areas contralateral to the anode including M1. Those results point toward rebalancing effects on age-dependent deterioration of interhemispheric inhibition (Vallesi et al., 2010), and the observed task-related dual effects can be interpreted as complex, synergistic bihemispheric modulations.
Table 1. Resting-state ECM analysis: results of paired $t$ tests

\begin{tabular}{|c|c|c|c|c|c|}
\hline Region & $\mathrm{k}_{\mathrm{E}}$ & $Z$ value & $x$ & $y$ & $z$ \\
\hline \multicolumn{6}{|l|}{ Dual versus sham } \\
\hline L middle frontal gyrus & 31 & 3.81 & -38 & 42 & 26 \\
\hline L superior/middle frontal gyrus & 33 & 3.43 & -17 & 18 & 50 \\
\hline L dorsal posterior cingulate cortex & 61 & 3.76 & 0 & -36 & 26 \\
\hline R frontal operculum/insula & 38 & -4.46 & 46 & -3 & 15 \\
\hline R parietal operculum/insula & 46 & -3.51 & 43 & -18 & 20 \\
\hline R hippocampus/parahippocampal gyrus & 44 & -4.45 & 17 & -24 & -10 \\
\hline Culmen (cerebellum) & 39 & -3.68 & 0 & -48 & -2 \\
\hline \multicolumn{6}{|l|}{ Anodal versus sham } \\
\hline L caudate nucleus & 41 & 4.71 & -17 & 27 & 9 \\
\hline L middle occipital/fusiform gyrus & 34 & 3.57 & -38 & -60 & -2 \\
\hline L middle/inferior frontal gyrus & 73 & 3.40 & -49 & 45 & 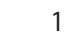 \\
\hline R precentral gyrus (somatosensory) & 45 & -3.78 & 17 & -27 & 64 \\
\hline R hippocampus/parahippocampal gyrus & 33 & -3.69 & 14 & -24 & -10 \\
\hline $\mathrm{R}$ middle/inferior temporal gyrus & 35 & -3.52 & 49 & -39 & 6 \\
\hline R precuneus & 87 & -3.47 & 6 & -66 & 28 \\
\hline \multicolumn{6}{|l|}{ Dual versus anodal } \\
\hline L dorsal posterior cingulate cortex & 35 & 3.46 & -6 & -30 & 31 \\
\hline Culmen (cerebellum) & 61 & -3.70 & -3 & -63 & -2 \\
\hline
\end{tabular}

Location and size of clusters with significantly increased (positive $Z$ values) or decreased (negative $Z$ values) Eigenvector centrality contrasting the $\mathrm{DCCS}$ conditions.

$\mathrm{k}_{\mathrm{F}}=$ cluster extent (number of voxels); $x-y-z=$ coordinates of peak voxels in Talairach space. 


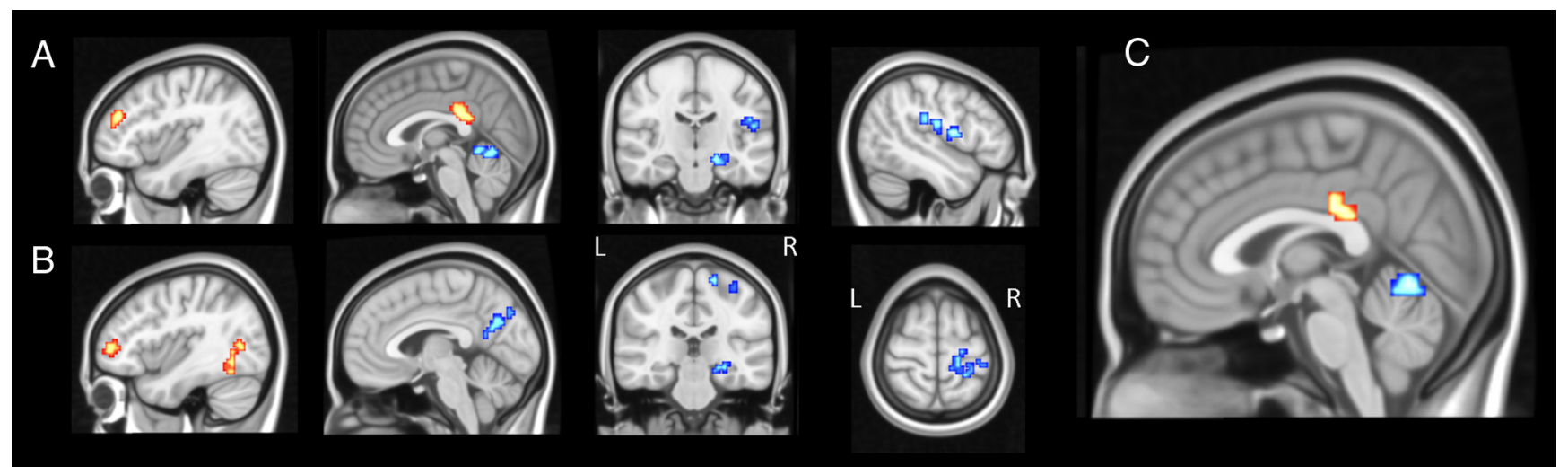

Figure 4. Eigenvector centrality mapping analysis. Paired $t$ tests of whole brain low-frequency fluctuation contrasting $(\boldsymbol{A})$ dual versus sham tDCS $[$ Talairach coordinates of $s$ lices are $x=-39 ; x=$ $-2 ; y=-20 ; x=45],(B)$ anodal versus sham $\operatorname{tDCS}[x=-39 ; x=4 ; y=-28 ; z=62]$, and $(C)$ dual versus anodal $\operatorname{tDCS}[x=0]$. Voxels were considered significant at $p<0.05$, corrected for multiple-comparisons using Monte-Carlo simulations. Clusters that exhibited enhanced ECM values are depicted in yellow to red ( $Z=0$ to $Z=4.71)$, those that exhibited decreased values in light to dark blue $(Z=0$ to $Z=-4.46)$.

Correlations between tDCS-induced laterality changes and microstructure of transcallosal M1 connections seen in our study further underline the importance of interhemispheric interplay. Interestingly, significant correlations between FA and changes in laterality were only observed for left tap, which fits well with the hypothesis that tDCS-induced effects on the M1 contralateral to the anode position are mediated by transcallosal fibers (Perez and Cohen, 2009). FA reductions have been linked to age-related white matter degeneration (Sullivan et al., 2010) also present in our group of older subjects (compare FA values with those of young healthy subjects) (Wahl et al., 2007), and more pronounced microstructural deterioration was associated with reduced effects of the stimulation (cf. Lindenberg et al., 2012b).

Two previous studies investigated task-related activity changes of unihemispheric tDCS using simultaneous fMRI and 2 min of continuous stimulation (Kwon and Jang, 2011) or on/off paradigms with even shorter durations (Antal et al., 2011). In our study, the task commenced after direct current had been applied constantly for $\sim 20$ min and continued throughout the task. Furthermore, we recruited older adults, whereas the previous investigations included younger subjects. Such methodological differences as well as heterogeneous tasks and fMRI designs render direct comparison difficult. During sham, task-related activity yielded intermediate values in between changes induced by dual and anodal tDCS. However, due to substantial interindividual variability (see SEM in Fig. 2), activity in M1 during sham tDCS was not significantly different from either of the active stimulation conditions, as described previously for unihemispheric tDCS (Antal et al., 2011). Therefore, it is even more remarkable that the comparison of unihemispheric and bihemispheric tDCS yielded highly significant results in this study.

A potential limitation of our study may be possible interactions between word-generation and subsequent choice reaction time tasks because cognitive and motor exercises have been shown to change M1 excitability differentially (Antal et al., 2007). However, we demonstrated that M1 activity was not influenced by tDCS during the language task participants underwent before performing the motor task. In addition, interactions would have been similar during the two active stimulation conditions in our study. Since in our main analysis of the choice reaction time task-related data, we directly compared those tDCS conditions with each other, it seems unlikely that potential interactions confounded this aspect of the results.

\section{Low-frequency oscillations}

The resting-state analysis demonstrated altered connectivity within a multimodal network using an approach that allows investigating spontaneous low-frequency oscillations across the entire brain without a priori hypotheses (Lohmann et al., 2010). Both anodal and dual tDCS yielded widespread polaritydependent network modulations. Compared with sham, various cortical and subcortical left-hemispheric areas (i.e., ipsilateral to the anode during both active tDCS conditions) showed increased ECM values, whereas a decrease was found in several righthemispheric regions (i.e., contralateral to the anode during both conditions and ipsilateral to the cathode during dual tDCS). Contrary, anodal tDCS applied to the left inferior frontal cortex yielded network-specific increases and decreases of ECM values in both hemispheres (Meinzer et al., 2012). Therefore, polaritydependent intrahemispheric modulations of low-frequency fluctuations cannot be considered a general effect of tDCS, but may be specific for M1 stimulation. More specifically, anodal and dual tDCS yielded a decrease of ECM values in right motor cortices compared with sham, indicating a functional decoupling of those areas. Similar effects have been described previously for cathodal tDCS using a seed-based approach (Polania et al., 2011a). As a complement, anodal tDCS has been shown to yield reductions in remote cortical connections of the target M1 using graphtheoretical analyses of resting-state data (Polania et al., 2011c). Notably, although unihemispheric anodal tDCS induced changes in the vicinity of the hand knob (i.e., homologous to the contralateral stimulation site) in our current study, dual tDCS effects were observed in opercular regions. Together with alterations of task-related activity and effects discussed below, this points toward more complex bihemispheric modulations caused by dual tDCS.

Previous resting-state fMRI studies demonstrated decreased functional connectivity of cingulate and premotor areas (Wu et al., 2007) and reduced interhemispheric inhibition in aging (Langan et al., 2010). In this context, tDCS-induced connectivity decreases in M1 and premotor areas of the right hemisphere and ipsilateral hippocampus (i.e., contralateral to the anode in both the dual and anodal tDCS condition), observed in our group of older subjects can be interpreted as reversal of age-related effects on interhemispheric connectivity. "Rebalancing" an imbalance in interhemispheric interplay has also been proposed to be an important feature of tDCS interventions after stroke (Schlaug et al., 2008). It provides the basis for downregulating the contral- 
esional M1 in combination with upregulating ipsilesional M1 spared by the lesion in chronic stroke patients (Lindenberg et al., 2010; Bolognini et al., 2011).

In addition to decreased connectivity in regions of the right hemisphere, an increase of ECM values could be observed in the contralateral rostral and dorsolateral prefrontal cortex, i.e., ipsilateral to the anode in both active stimulation conditions. This region is part of a "frontoparietal control network" that links default mode and attention networks (Spreng et al., 2013), so that tDCS-induced modulations can be interpreted as a networkindependent cross-modal effects.

Most strikingly, however, dual tDCS resulted in significantly higher ECM values in the left cingulate cortex compared with sham or anodal tDCS. The cluster comprised the posterior border of the caudal cingulate motor area and extended into the dPCC. This latter region is tightly connected with the cingulate motor areas, which in turn project to premotor cortices (Vogt et al., 2006). Hence, our data indicate that the concurrent modulation of bihemispheric M1 excitability yields a strengthening of the dPCC as a major hub that "regulates skeletomotor functions" (Vogt et al., 2006), a unique feature of dual tDCS.

With physiological aging the PCC becomes less connected structurally (Zhu et al., 2012) and functionally (Andrews-Hanna et al., 2007), a finding even more prominent in neurodegenerative dementia (Greicius et al., 2004). Therefore, future studies may explore potential beneficial dual tDCS effects on cognition as well.

\section{Conclusions}

This study suggests that both anodal and dual tDCS can potentially be used to counteract age-related impairment of interhemispheric interactions. Unihemispheric lesions of the motor system yield an even more pronounced imbalance of interhemispheric interactions (Murase et al., 2004), which explains the beneficial effects of unihemispheric (Hummel et al., 2005) or bihemispheric tDCS (Lindenberg et al., 2010) on motor recovery after stroke. Differential effects of bihemispheric compared with unihemispheric stimulation may not merely be mediated by a "simple" add-on effect of anodal and cathodal stimulation, but rather due to synergistic bihemispheric network modulations involving the left dPCC, a major hub mediating motor control.

\section{References}

Alon G, Roys SR, Gullapalli RP, Greenspan JD (2011) Non-invasive electrical stimulation of the brain (ESB) modifies the resting-state network connectivity of the primary motor cortex: a proof of concept fMRI study. Brain Res 1403:37-44. CrossRef Medline

Andrews-Hanna JR, Snyder AZ, Vincent JL, Lustig C, Head D, Raichle ME, Buckner RL (2007) Disruption of large-scale brain systems in advanced aging. Neuron 56:924-935. CrossRef Medline

Antal A, Terney D, Poreisz C, Paulus W (2007) Towards unravelling taskrelated modulations of neuroplastic changes induced in the human motor cortex. Eur J Neurosci 26:2687-2691. CrossRef Medline

Antal A, Polania R, Schmidt-Samoa C, Dechent P, Paulus W (2011) Transcranial direct current stimulation over the primary motor cortex during fMRI. Neuroimage 55:590-596. CrossRef Medline

Baudewig J, Nitsche MA, Paulus W, Frahm J (2001) Regional modulation of BOLD MRI responses to human sensorimotor activation by transcranial direct current stimulation. Magn Reson Med 45:196-201. CrossRef Medline

Behrens TE, Berg HJ, Jbabdi S, Rushworth MF, Woolrich MW (2007) Probabilistic diffusion tractography with multiple fibre orientations: what can we gain? Neuroimage 34:144-155. CrossRef Medline

Bolognini N, Vallar G, Casati C, Latif LA, El-Nazer R, Williams J, Banco E, Macea DD, Tesio L, Chessa C, Fregni F (2011) Neurophysiological and behavioral effects of tDCS combined with constraint-induced movement therapy in poststroke patients. Neurorehabil Neural Repair 25:819-829. CrossRef Medline

Bonacich P (2007) Some unique properties of eigenvector centrality. Soc Networks 29:555-564. CrossRef

Eickhoff SB, Stephan KE, Mohlberg H, Grefkes C, Fink GR, Amunts K, Zilles K (2005) A new SPM toolbox for combining probabilistic cytoarchitectonic maps and functional imaging data. Neuroimage 25:1325-1335. CrossRef Medline

Greicius MD, Srivastava G, Reiss AL, Menon V (2004) Default-mode network activity distinguishes Alzheimer's disease from healthy aging: evidence from functional MRI. Proc Natl Acad Sci U S A 101:4637-4642. CrossRef Medline

Hummel F, Celnik P, Giraux P, Floel A, Wu WH, Gerloff C, Cohen LG (2005) Effects of non-invasive cortical stimulation on skilled motor function in chronic stroke. Brain 128:490-499. CrossRef Medline

Klöppel S, van Eimeren T, Glauche V, Vongerichten A, Münchau A, Frackowiak RS, Büchel C, Weiller C, Siebner HR (2007) The effect of handedness on cortical motor activation during simple bilateral movements. Neuroimage 34:274-280. CrossRef Medline

Kwon YH, Jang SH (2011) The enhanced cortical activation induced by transcranial direct current stimulation during hand movements. Neurosci Lett 492:105-108. CrossRef Medline

Langan J, Peltier SJ, Bo J, Fling BW, Welsh RC, Seidler RD (2010) Functional implications of age differences in motor system connectivity. Front Syst Neurosci 4:17. CrossRef Medline

Lauritzen M, Mathiesen C, Schaefer K, Thomsen KJ (2012) Neuronal inhibition and excitation, and the dichotomic control of brain hemodynamic and oxygen responses. Neuroimage 62:1040-1050. CrossRef Medline

Lindenberg R, Renga V, Zhu LL, Nair DN, Schlaug G (2010) Bihemispheric brain stimulation facilitates motor recovery in chronic stroke patients. Neurology 75:2176-2184. CrossRef Medline

Lindenberg R, Zhu LL, Schlaug G (2012a) Combined central and peripheral stimulation to facilitate motor recovery after stroke: the effect of number of sessions on outcome. Neurorehabil Neural Repair 26:479-483. CrossRef Medline

Lindenberg R, Zhu LL, Rüber T, Schlaug G (2012b) Predicting functional motor potential in chronic stroke patients using diffusion tensor imaging. Hum Brain Mapp 33:1040-1051. CrossRef Medline

Lohmann G, Margulies DS, Horstmann A, Pleger B, Lepsien J, Goldhahn D, Schloegl H, Stumvoll M, Villringer A, Turner R (2010) Eigenvector centrality mapping for analyzing connectivity patterns in fMRI data of the human brain. PLoS One 5:e10232. CrossRef Medline

Meinzer M, Antonenko D, Lindenberg R, Hetzer S, Ulm L, Avirame K, Flaisch T, Flöel A (2012) Electrical brain stimulation improves cognitive performance by modulating functional connectivity and task-specific activation. J Neurosci 32:1859-1866. CrossRef Medline

Mordillo-Mateos L, Turpin-Fenoll L, Millán-Pascual J, Núñez-Pérez N, Panyavin I, Gómez-Argüelles JM, Botia-Paniagua E, Foffani G, Lang N, Oliviero A (2012) Effects of simultaneous bilateral tDCS of the human motor cortex. Brain Stimul 5:214-222. CrossRef Medline

Murase N, Duque J, Mazzocchio R, Cohen LG (2004) Influence of interhemispheric interactions on motor function in chronic stroke. Ann Neurol 55:400-409. CrossRef Medline

Nichols T, Brett M, Andersson J, Wager T, Poline JB (2005) Valid conjunction inference with the minimum statistic. Neuroimage 25:653-660. CrossRef Medline

Nitsche MA, Paulus W (2000) Excitability changes induced in the human motor cortex by weak transcranial direct current stimulation. J Physiol 527:633-639. CrossRef Medline

Nitsche MA, Paulus W (2001) Sustained excitability elevations induced by transcranial DC motor cortex stimulation in humans. Neurology 57: 1899-1901. CrossRef Medline

Nitsche MA, Paulus W (2011) Transcranial direct current stimulation: update 2011. Restor Neurol Neurosci 29:463-492. CrossRef Medline

Nitsche MA, Fricke K, Henschke U, Schlitterlau A, Liebetanz D, Lang N, Henning S, Tergau F, Paulus W (2003) Pharmacological modulation of cortical excitability shifts induced by transcranial direct current stimulation in humans. J Physiol 553:293-301. CrossRef Medline

Oldfield RC (1971) The assessment and analysis of handedness: the Edinburgh inventory. Neuropsychologia 9:97-113. CrossRef Medline

Perez MA, Cohen LG (2009) Interhemispheric inhibition between primary 
motor cortices: what have we learned? J Physiol 587:725-726. CrossRef Medline

Polanía R, Nitsche MA, Paulus W (2011a) Modulating functional connectivity patterns and topological functional organization of the human brain with transcranial direct current stimulation. Hum Brain Mapp 32: 1236-1249. CrossRef Medline

Polanía R, Paulus W, Nitsche MA (2011b) Modulating cortico-striatal and thalamo-cortical functional connectivity with transcranial direct current stimulation. Hum Brain Mapp 33:2499-2508. CrossRef Medline

Polanía R, Paulus W, Antal A, Nitsche MA (2011c) Introducing graph theory to track for neuroplastic alterations in the resting human brain: a transcranial direct current stimulation study. Neuroimage 54:2287-2296. CrossRef Medline

Reis J, Schambra HM, Cohen LG, Buch ER, Fritsch B, Zarahn E, Celnik PA, Krakauer JW (2009) Noninvasive cortical stimulation enhances motor skill acquisition over multiple days through an effect on consolidation. Proc Natl Acad Sci U S A 106:1590-1595. CrossRef Medline

Rüber T, Schlaug G, Lindenberg R (2012) Compensatory role of the corticorubro-spinal tract in motor recovery after stroke. Neurology 79:515-522. CrossRef Medline

Salvador R, Suckling J, Schwarzbauer C, Bullmore E (2005) Undirected graphs of frequency-dependent functional connectivity in whole brain networks. Philos Trans R Soc Lond B Biol Sci 360:937-946. CrossRef Medline

Schlaug G, Renga V, Nair D (2008) Transcranial direct current stimulation in stroke recovery. Arch Neurol 65:1571-1576. CrossRef Medline

Seghier ML (2008) Laterality index in functional MRI: methodological issues. Magn Reson Imaging 26:594-601. CrossRef Medline

Smith SM, Jenkinson M, Woolrich MW, Beckmann CF, Behrens TE, Johansen-Berg H, Bannister PR, De Luca M, Drobnjak I, Flitney DE, Niazy RK, Saunders J, Vickers J, Zhang Y, De Stefano N, Brady JM, Matthews PM (2004) Advances in functional and structural MR image analysis and implementation as FSL. Neuroimage 23:S208-219. CrossRef Medline

Spreng RN, Sepulcre J, Turner GR, Stevens WD, Schacter DL (2013) Intrin- sic architecture underlying the relations among the default, dorsal attention, and frontoparietal control networks of the human brain. J Cogn Neurosci 25:74-86. CrossRef Medline

Stagg CJ, O'Shea J, Kincses ZT, Woolrich M, Matthews PM, Johansen-Berg H (2009) Modulation of movement-associated cortical activation by transcranial direct current stimulation. Eur J Neurosci 30:1412-1423. CrossRef Medline

Sullivan EV, Rohlfing T, Pfefferbaum A (2010) Quantitative fiber tracking of lateral and interhemispheric white matter systems in normal aging: relations to timed performance. Neurobiol Aging 31:464-481. CrossRef Medline

Taubert M, Lohmann G, Margulies DS, Villringer A, Ragert P (2011) Longterm effects of motor training on resting-state networks and underlying brain structure. Neuroimage 57:1492-1498. CrossRef Medline

Vallesi A, McIntosh AR, Kovacevic N, Chan SC, Stuss DT (2010) Age effects on the asymmetry of the motor system: evidence from cortical oscillatory activity. Biol Psychol 85:213-218. CrossRef Medline

Vines BW, Cerruti C, Schlaug G (2008) Dual-hemisphere tDCS facilitates greater improvements for healthy subjects' non-dominant hand compared with uni-hemisphere stimulation. BMC Neurosci 9:103. CrossRef Medline

Vogt BA, Vogt L, Laureys S (2006) Cytology and functionally correlated circuits of human posterior cingulate areas. Neuroimage 29:452-466. CrossRef Medline

Wahl M, Lauterbach-Soon B, Hattingen E, Jung P, Singer O, Volz S, Klein JC, Steinmetz H, Ziemann U (2007) Human motor corpus callosum: topography, somatotopy, and link between microstructure and function. J Neurosci 27:12132-12138. CrossRef Medline

Wu T, Zang Y, Wang L, Long X, Hallett M, Chen Y, Li K, Chan P (2007) Aging influence on functional connectivity of the motor network in the resting state. Neurosci Lett 422:164-168. CrossRef Medline

Zhu W, Wen W, He Y, Xia A, Anstey KJ, Sachdev P (2012) Changing topological patterns in normal aging using large-scale structural networks. Neurobiol Aging 33:899-913. CrossRef Medline 